\title{
Differential expression of heat shock protein genes and their splice variants in bovine preimplantation embryos
}

\author{
B. Zhang, ${ }^{*} \dagger$ F. Peñagaricano, $†$ A. Driver, $\dagger$ H. Chen, ${ }^{*} \ddagger$ and H. Khatibt ${ }^{1}$ \\ ${ }^{*}$ College of Animal Science and Technology, Northwest A\&F University, Yangling, Shaanxi, 712100, China \\ †Department of Dairy Science, University of Wisconsin-Madison 53706 \\ łInstitute of Cellular and Molecular Biology, Xuzhou Normal University, Xuzhou, Jiangsu, 221116, China
}

\section{ABSTRACT}

Heat shock proteins (HSP) are among the first proteins produced during embryonic development and are crucial to cell function; their activities include folding, unfolding, transport, and localization of proteins and differentiation and regulation of the embryonic cell cycle. Although HSP have been extensively studied in humans and mice, limited information exists on the roles of these genes in bovine embryos. As such, the objectives of this study were to profile the expression of HSP and their splice variants in bovine embryos (degenerates vs. blastocysts) and to carry out association analysis with fertility traits. Quantitative real time PCR analysis revealed differences in expression that ranged from 1.5- to 7.6-fold in degenerate embryos compared with blastocysts. Interestingly, all members of the HSP40 family were found to be upregulated in degenerate embryos. Analysis of current bovine Ensembl data showed that 13 HSP genes have 1 transcript each, 2 genes have 2 transcripts each, and 2 have 3 transcripts each. Some splice variants show differential expression between degenerate embryos and blastocysts, whereas others were not expressed at all in embryos, which implies different functions of these transcripts in embryonic development. The most significant differentially expressed genes were further investigated for association with fertility and development traits. Single nucleotide polymorphisms in DNAJC15 and DNAJC27 were found to be associated with blastocyst rate and fertilization rate, respectively. Collectively, the roles of HSP in fertilization and early development of cattle embryos are important.

Key words: heat shock protein, bovine, embryo, alternative splicing

\section{INTRODUCTION}

Heat shock proteins (HSP) are among the most highly conserved proteins in nature and have been

Received December 28, 2010.

Accepted April 21, 2011.

${ }^{1}$ Corresponding author: hkhatib@wisc.edu found in all organisms studied from bacteria to humans (Becker and Craig, 1994). The structure and roles of HSP as molecular chaperones in folding, transport, and assembly of proteins, as well as in protecting the cell under different stress conditions, have been extensively studied and reported in the scientific literature (Becker and Craig, 1994; Nollen and Morimoto, 2002; Qiu et al., 2006). The fact that HSP are essential in the folding, stability, and cellular localization of newly synthesized proteins implies key roles of these proteins in apoptosis, cell differentiation, and regulation of the embryo cell cycle (Luft and Dix, 1999; Lanneau et al., 2007). Strong evidence that has accumulated on the expression of HSP during spermatogenesis, oogenesis, and embryogenesis suggests they have important functions in fertilization and during the preimplantation period (Neuer et al., 1999). Mouse embryos cultured with monoclonal antibodies to HSP have been found to display a significantly decreased blastocyst rate (Neuer et al., 1998). Al-Katanani and Hansen (2002) reported that the addition of antibodies for the induced form of HSP70 decreased the blastocyst rate of cattle embryos, suggesting that HSP70 is involved in proper embryonic development. Matwee and colleagues (2001) showed that fertilization - measured as the number of spermatozoa tightly bound to a zona pellucida - and embryo development in cattle were significantly affected by the presence of different concentrations of anti-HSP70 antibodies.

Although strong evidence exists in the literature on the roles of HSP in early embryonic development, most of the studies have focused on the mouse and only a few HSP genes have been studied in cattle embryos. Given that HSP are among the first proteins produced during embryonic development (Neuer et al., 1999), determining expression patterns of these genes in developed versus arrested embryos could lead to the identification of specific genes involved in reproductive success. This is of particular interest in cattle because of decreasing fertility over the past few decades (Dobson et al., 2007; Leroy et al., 2008). Furthermore, recent studies have shown that low fertilization rates and embryonic loss seem to be the main factors in dairy cattle infertil- 
ity (Santos et al., 2004; Morris and Diskin, 2008). To characterize genetic factors involved in embryonic loss, and hence infertility, an in-vitro fertilization (IVF) experimental system in cattle has been used to associate candidate genes and pathways with fertilization rate and embryonic survival at both the genomic and gene expression levels (Khatib et al., 2008a,b, 2009; Huang et al., 2010a,b; Huang and Khatib, 2010).

The present study hypothesizes that the high structural and functional conservation of HSP during evolution suggests crucial roles in fertilization, embryo development, and thus, fertility in cattle. Also, some HSP are considered housekeeping genes that are essential for many cell functions. Hence, the objectives of this study were to evaluate the expression levels of 17 HSP genes in biological replicate bovine embryo pools that differ in their morphology and developmental statuses and to look for associations of polymorphisms in significant genes with fertility traits. In addition, recent studies have shown that more than $90 \%$ of the human multiexon genes encode multiple transcripts (Wang et al., 2008) and that alternative splicing is considered to be a key factor in the evolution of phenotypic complexity (Blencowe, 2006). However, limited information exists on the pattern of alternative splicing in cattle or its roles in embryonic development. Recently, using highthroughput sequencing of transcriptomes (RNA-seq), thousands of alternative splicing events were detected and tested for their association with the status of embryonic development (Huang and Khatib, 2010). Thus, another objective of this study was to compare the expression levels of splice variants of HSP in degenerate embryos and healthy blastocysts.

\section{MATERIALS AND METHODS}

\section{In Vitro Maturation, Fertilization, and Embryo Culture}

In this study, fertilization and embryo production were performed in 2 different experiments. The first experiment is a comparison of expression profiles of 17 HSP between 2 populations of embryos differing in their morphology, and the second experiment is a genomic association analysis of genes-found to be differentially-expressed in the first experiment-with fertilization and blastocyst rates.

\section{Experiment l: Expression Analysis of HSP Genes in Cattle Embryos}

Ovaries were obtained from a local abattoir and processed for IVF using standard protocol (Khatib et al., 2008b; Huang et al., 2010b). Briefly, oocytes were aspirated from 2 to 6 -mm follicles and washed in Tyrode's albumin lactate pyruvate (TALP)-Hepes medium, after which they underwent maturation in supplemented M199 media (Khatib et al., 2008b). Incubation was completed at $5 \% \mathrm{CO}_{2}$ in air at $39^{\circ} \mathrm{C}$ and high humidity for 22 to $24 \mathrm{~h}$, at which time oocytes were then washed in TALP-Hepes medium and moved into fresh IVF-TALP medium (BioWhittaker Inc., Walkersburg, MD). Semen samples from 2 different bulls were used for fertilization; sperm underwent Percoll separation (45-90\% gradient) and were adjusted to a final concentration of $1 \times 10^{6} / \mathrm{mL}$ (Parrish et al., 1995). Fertilization was marked as d 0 of development and completed by combining sperm, heparin, and penicillamine, hypotaurine, and epinephrine with the oocytes in $44-\mu \mathrm{L}$ drops of media (Khatib et al., 2008b). Incubation was then continued for 22 to $24 \mathrm{~h}$, after which the putative zygotes were washed in TALP-Hepes medium, denuded of remaining cumulus cell complexes, and placed into supplemented synthetic oviductal fluid (BioWhittaker Inc.) and returned to incubation (Khatib et al., 2008b).

\section{Morphological Assessment}

Putative zygotes were cultured over an 8-d period, during which assessments of their developmental progress were made. By d 5 of development, a bovine embryo should attain approximately 16 to 32 cells and show signs of cellular compaction deeming it as morula. Embryos failing to show these characteristics were assumed to have been arrested in development and were excluded from analysis. Morulas were then continued in culture until d 8. By this time, an embryo should show evidence of a fluid-filled cavity (blastocoele), which gives rise to the differentiation of the inner cell mass and the trophectoderm, qualifying it as a blastocyst. For expression analysis, 2 populations of embryos were collected. The first consisted of embryos that attained a compacted morula status by d 5 but failed to form a blastocoele by d 8, referred as degenerate embryos. The second consisted of embryos that developed into blastocysts by $\mathrm{d} 8$. Embryos from each morphological group were collected in pools of 20 and preserved in RNAlater (Ambion Inc., Austin, TX). Two bulls were used with 2 sets of biological replicate pools (total of 4 biological replicates) from each to prevent maternal crossover.

\section{Quantitative Real-Time PCR}

A previous study in our laboratory using microarray expression analysis has revealed that many genes are differentially expressed between blastocysts and degenerate embryos (Huang et al., 2010b). Seventeen HSP genes that showed 1.5-fold or higher differences in expression between the embryo groups described in the 
Table 1. Primers used for total expression and alternative splicing analysis of heat shock protein (HSP) genes using quantitative real-time PCR (qRT-PCR) and RT-PCR

\begin{tabular}{|c|c|c|c|c|}
\hline Item & Family $^{1}$ & Forward primer $5^{\prime}-3^{\prime}$ & Reverse primer $5^{\prime}-3^{\prime}$ & $\begin{array}{c}\text { Amplicon } \\
\text { size (bp) }\end{array}$ \\
\hline \multicolumn{5}{|l|}{ Gene } \\
\hline GAPDH & & TGCCCAGAATATCATCCC & AGGTCAGATCCACAACAG & 134 \\
\hline DNAJC15 & HSP40 & AGGTCGCTACGCATTTCAG & GACTTGCTTCTCGCCTACTC & 137 \\
\hline DNAJC19 & HSP40 & GGACTGACCATTGCTGCTG & CAAACCCACCTCTGTAATAGC & 140 \\
\hline DNAJC24 & HSP40 & GAAATATGGGACCAGTAGATGC & TGTAACTTCTTCTGCTTCATCC & 134 \\
\hline HSPA9 & HSP70 & GACCAACTGCCTGCTGATG & GATGCCGCCTGCCTTATG & 113 \\
\hline HSPA 8 & HSC70 & CGCAGAAGCCTACCTTGG & GTTGAGACCAGCAATAGTTCC & 115 \\
\hline HSPA14 & HSP70 & AACCTTAGCACAGTACCTAGC & TGTCAGCACCGTTCATCAG & 101 \\
\hline$H S B P 1$ & HSBP & CATGTCCGACCAGATCATTG & TTCACTGTCCAGCTCTTCC & 115 \\
\hline HSPE1 & HSP10 & GCAAGCAACGGTGGTAGC & ACTTTGGTGCCTCCATATTCTG & 117 \\
\hline DNAJB1 & HSP40 & GAGGAGAAGTTCAAGGAGATCG & TTAGTACCGCCGCTGCTC & 134 \\
\hline DNAJB12 & HSP40 & GCAAACTAGCCCTCAAATTCC & CCTTGTCATCACCGAACTGG & 141 \\
\hline \multicolumn{5}{|l|}{ Transcript } \\
\hline DNAJC5-667 & & AGGAGACGGAGTTCTATG & CACGTTCACACCTCAAC & 126 \\
\hline DNAJC5-725 & & GGCCCTGTTCATCTTCTG & GGCACAGACCCTCTCAT & 181 \\
\hline DNAJC5-1049 & & GGGTTCAACTAAATCCAGGA & ACGCCATCTCTGTGACTA & 79 \\
\hline DNAJC19-475 & & GGATCTCCTTATATAGCAGCCAAA & AGCCTTCCСTCCCAGTGA & 93 \\
\hline DNAJC19-536 & & ATGCTCATCGGCGAATTATG & AGCTGGAACGCATAAGAGAA & 161 \\
\hline DNAJC24-628 & & CTCATTTTAATGGAAGATG & GTATCACAAGAAATCAGT & 176 \\
\hline DNAJC24-2172 & & CATCCAGATAAACAGAGT & GGTCCCATATTTCTTAGAT & 161 \\
\hline DNAJC24-1893 & & CAAAAGAAAGTATCTCATTC & TAACTTCTTCTGCTTCATC & 176 \\
\hline DNAJB12-1220 & & ACCGACTGTCAGAGACTATG & CGGCCTCCAATTCCATTT & 127 \\
\hline
\end{tabular}

${ }^{1} \mathrm{HSC}=$ heat shock constitutive protein; HSBP $=$ heat shock factor binding protein.

study of Huang et al. (2010b) were chosen for validation of differential expression and further investigation in new sets of blastocysts and degenerate embryo pools using quantitative real-time PCR (qRT-PCR) analysis. Primers (Table 1) were designed to amplify fragments spanning more than one exon to exclude the possibility of genomic DNA contamination in the qRT-PCR reactions using the Beacon Designer software (Premier Biosoft International, Palo Alto, CA). Total RNA was extracted from pools of embryos using RNAqueousMicro (Ambion Inc.) and quality controlled using a RNA 6000 Pico Chip (Agilent Technologies Inc., Santa Clara, CA). Messenger RNA was amplified using MessageAmp II (Ambion Inc.), followed by cDNA synthesis using iScript (Bio-Rad Laboratories Inc., Hercules, CA) according to manufacturers' instructions. Reactions of qRT-PCR were run on a DNA Engine Opticon 2 Detection System (MJ Research Inc., Watertown, MA) using iQ SYBR Green Supermix kit (Bio-Rad Laboratories Inc.). Each sample was run in quadruplicate and all 4 expression results were averaged. Analysis of gene expression levels was conducted using the $2^{-\Delta \Delta \mathrm{Ct}}$ (where $\mathbf{C t}$ is the cycle threshold) method (Livak and Schmittgen, 2001). The selection of the housekeeping gene
$G A P D H$ as an endogenous control was as described in Huang et al. (2010b).

\section{Experiment II: Association Analysis of Differentially Expressed HSP with Fertilization and Blastocyst Rates}

To further investigate the involvement in fertility of genes (DNAJC15, DNAJC19, DNAJC24, and $D N A J C 27$ ) that showed the highest-fold change in expression in degenerate embryos versus blastocysts, an association analysis between SNP in these genes and 2 main fertility traits, fertilization rate and blastocyst rate, was performed. Using the IVF experimental system, several genes were found to be significantly associated with variation in fertilization and embryonic survival rates (Khatib et al., 2008a,b; 2009).

Phenotypic Data. To generate phenotypic data for association analysis, a total of 6,893 IVF were performed using oocytes from 399 ovaries (obtained from 399 Holstein cows) and semen samples from 12 Holstein bulls. For 92 ovaries, oocytes were fertilized by 2 different bulls each. Fertilization rate was calculated as the number of cleaved embryos $48 \mathrm{~h}$ postfertilization out 
of the total number of oocytes exposed to sperm. Blastocyst rate was calculated as the number of embryos that reached blastocyst stage out of the total embryos cultured by d 8 .

Genotyping. The DNA was extracted from ovaries (n = 399) using standard phenol/chloroform protocols (Sambrook and Russell, 2001). The DNA concentrations were measured using a spectrophotometer (Ultraspec 2100; Amersham Biosciences, Uppsala, Sweden). For polymorphism identification, 3 DNA pools were constructed from 20 different ovary/cow samples to contain $25 \mathrm{ng}$ of DNA from each sample. The DNA pools were amplified with different sets of primers designed from the coding and $5^{\prime}$ and $3^{\prime}$ untranslated regions (UTR) of the 4 candidate genes (Supplementary Table 1). Amplification, sequencing of PCR products, and SNP identification were as described in Khatib et al. (2008a,b). Single nucleotide polymorphisms identified in DNAJC15, DNAJC19, DNAJC24, and DNAJC27 were genotyped for the 399 ovary/cow samples at GeneSeek Inc. (Lincoln, NE).

\section{Statistical Analysis}

For expression analysis, normalized gene expression values $(\Delta \mathrm{Ct})$ were analyzed using a general linear model including the fixed effects of the bull, the type of embryo (blastocyst or degenerate embryo), and the random effect of the pool. Association between the normalized gene expression and the type of embryo was tested using a likelihood ratio test by comparing this model to a reduced model without the embryo effect. The mean and the range of the fold change for each gene were calculated as $2^{-\Delta \Delta \mathrm{Ct}}$, using the estimated $\Delta \Delta \mathrm{Ct}$ value \pm standard error.

For genomic association analysis between SNP and fertilization and blastocyst rates, the following mixed linear model was used:

$$
y_{i j k}=\mu+o_{i}+b_{j}+S N P_{i j k}+e_{i j k},
$$

where $y_{i j k}$ represents, in turn, the fertilization or survival rate of oocytes $k$ from ovary $i$ fertilized with semen from bull $j ; \mu$ represents a general mean for the trait considered; $o_{i}$ represents the random effect of the individual ovary from which oocytes were harvested; $b_{j}$ represents the random effect of the sire used in the fertilization; $S N P_{i j k}$ represents the fixed effect of the genotypic class for the SNP considered; and $e_{i j k}$ represents the residuals, assumed normal, independent, and identically distributed with mean 0 and variance $\mathbf{I} \sigma_{e}^{2}$. Ovaries and bulls were assumed uncorrelated with variance structures $\mathbf{I} \sigma_{o}^{2}$ and $\mathbf{I} \sigma_{b}^{2}$, respectively. The associa- tion between the SNP and fertilization or blastocyst rates was tested using a likelihood ratio test by comparing the full model to a reduced model without the SNP effect. All analyses were performed using the lme4 package of $\mathrm{R}$ language/environment ( $\mathrm{R}$ Development Core Team, 2009).

\section{RESULTS}

\section{Expression Profiling of HSP Genes in Embryos}

Expression differences between degenerate embryos and blastocysts were estimated for 17 HSP genes in 4 sets of biological replicates using qRT-PCR (Figure 1). A range of 1.5- to 7.6-fold difference in expression was observed between embryo groups. Interestingly, all HSP40 gene family members were found to be upregulated in degenerate embryos compared with blastocysts (Figure 1). For example, DNAJC15 $(P<0.0001)$, DNAJC19 $(P<0.0001)$, DNAJC24 $(P=0.002)$, and DNAJC27 $(P=0.0098)$ showed an average of 7.6-, 4.8-, $3.3-, 4.33$-fold differences in expression, respectively. In contrast, only 2 members of the HSP70 family (HSPA14 and $H S P A 9)$ showed upregulation in degenerate embryos, whereas 4 HSP70 members (HSPA2, HSPA4, HSPA5, and HSPA8) showed higher expression levels in blastocysts than in degenerate embryos (Figure 1).

\section{Differential Expression of Alternatively Spliced Transcripts}

Splice variants of HSP genes were identified using the genebuild procedure of Ensembl (http://uswest. ensembl.org). In this procedure, annotation of transcripts is based on mRNA and protein sequences deposited in public scientific databases. An Ensembl search revealed 1 transcript per gene for 13 genes, 2 transcripts each for DNAJB12 and DNAJC19, and 3 transcripts each for DNAJC5 and DNAJC24. Expression levels of splice variants of DNAJC5, DNAJC19, DNAJC24, and DNAJB12 (Figure 2) were estimated in 4 sets of biological replicates of blastocysts and degenerate embryos using qRT-PCR. Transcripts DNAJC5-1049 and DNAJC5-667 (Figure 2) showed 2.53- and 1.67-fold higher expression, respectively, in degenerate embryos compared with blastocysts, whereas the DNAJC5-725 transcript was not detected in any of the embryo groups. For DNAJC19, only transcript DNAJC19-536 was detected in embryos. For DNAJC24, the DNAJC24-2172 transcript showed a 3.22-fold higher expression in degenerate embryos compared with blastocysts, whereas transcript DNAJC24-628 was lowly expressed in all embryo samples. The DNAJC24-1893 transcript could not be detected in embryos. The sequences of 


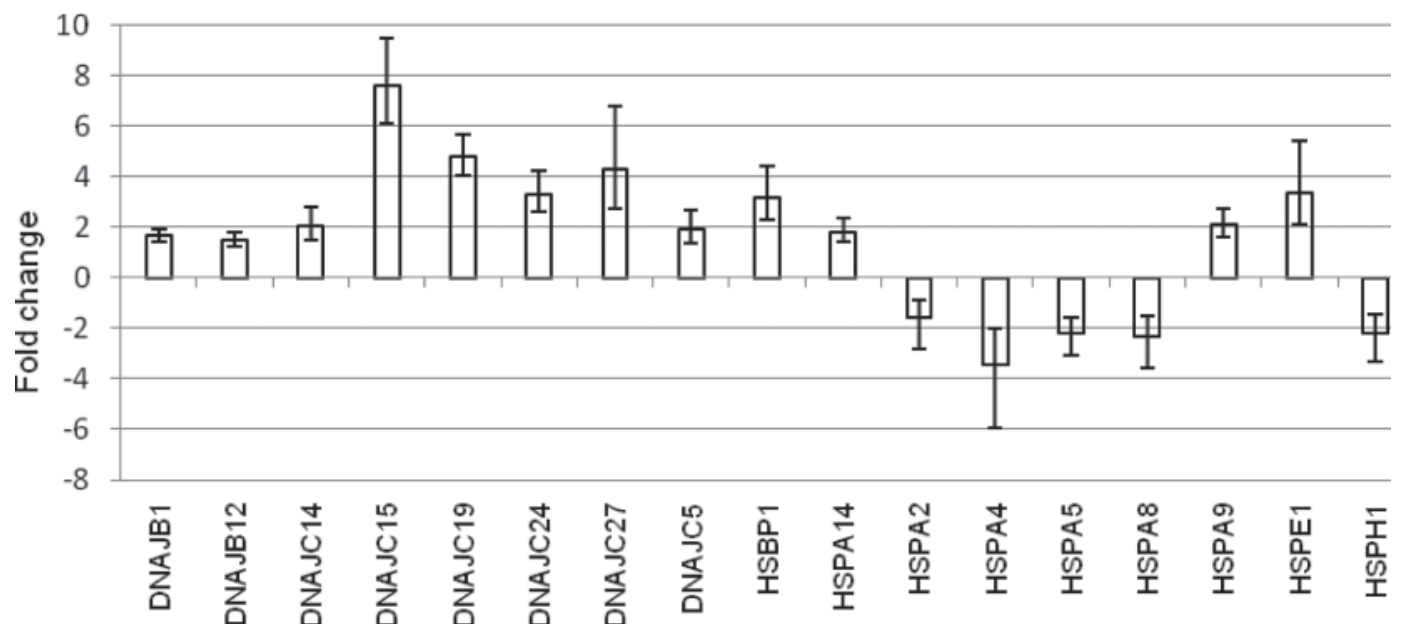

Figure 1. Fold change differences in expression of heat shock protein genes in bovine embryos using quantitative real-time PCR (qRT-PCR). Data are shown as mean +/- maximum- and minimum-fold changes. Upregulation in degenerate and blastocyst embryos is represented by bars above and below, respectively, the x-axis. The qRT-PCR was performed in 4 sets of biological replicates of blastocysts and degenerate embryos.

the 2 DNAJB12 transcripts were overlapping so that only transcript DNAB12-1220 could be amplified using transcript-specific primers. The DNAB12-1220 transcript showed a 1.95-fold higher expression in degenerate embryos versus blastocysts.

\section{Association of HSP Polymorphisms with Fertilization and Blastocyst Rates}

Single nucleotide polymorphisms in genes DNAJC15, DNAJC19, DNAJC24, and DNAJC27 were tested for association with fertilization and blastocyst rates. These genes showed the highest-fold differences in expression between embryo groups (Figure 1) and the highest statistical significance in the general linear model analysis $(P<0.01)$. Using the pooled DNA sequencing approach, 5 SNP were identified in the $3^{\prime}$ UTR of DNAJC15, 1 SNP in exon 5 of DNAJC19, 1 SNP and one 4-bp deletion in the 3' UTR of DNAJC24, and $3 \mathrm{SNP}$ in the $3^{\prime} \mathrm{UTR}$ of DNAJC2\%. All SNP in each of the DNAJC15 and DNAJC27 genes showed high linkage disequilibrium with each other (Figure 3). Polymorphisms in DNAJC19 and DNAJC24 did not show significant associations with fertility traits. Table 2 shows fertilization and blastocyst rates for the genotypic classes of the DNAJC15 and DNAJC2\% SNP. For DNAJC27 SNP36016, oocytes collected from genotype GG ovaries showed a $69.3 \%$ fertilization rate versus $62.2 \%$ for oocytes collected from $\mathrm{CC}$ ovaries $(P$ $=0.034$ ). For DNAJC15 SNP85146, the blastocyst rate of embryos produced from GG dams was 40.1 vs. 31.0 and $28.1 \%$ for embryos produced from $\mathrm{AA}$ and $\mathrm{AG}$ dams, respectively (Table 2).

\section{DISCUSSION}

The HSP are highly conserved across most organisms; therefore, it is conceivable to hypothesize that they play a vital role in survival and development. The objective of this study was to investigate the roles of HSP genes in early embryonic development and fertility in cattle at both genomic and gene expression levels. A total of 17 candidate genes were chosen based on differential expression results observed in a previous microarray analysis of bovine embryos. To test whether or not these genes have roles in early development of cattle embryos, their expression levels were quantified and compared between blastocysts and degenerate embryos. All 17 genes showed expression differences that ranged between 1.5- and 7.6-fold between the embryo groups.

All HSP40 family genes were found to be upregulated in degenerate embryos versus blastocysts. Although the specific functions of HSP40 have not been reported in cattle, studies from other species have shown that these genes play important roles in protecting cells under stress conditions (Lanneau et al., 2007). It is well established that ATP hydrolysis is necessary for protein-folding activity of HSP70 and that HSP40 stimulate ATPase activity and stabilize the interaction of HSP70 with their substrates (Qiu et al., 2006). Gotoh and colleagues (2004) reported that HSP40 combine with HSP70 to act as chaperones to protect cells from apoptosis. Degenerate embryos in the IVF system are growth-arrested embryos and seem to undergo partial apoptosis. Research has shown that HSP are upregulated to prevent apoptosis triggered by different stimuli 
DNAJC5
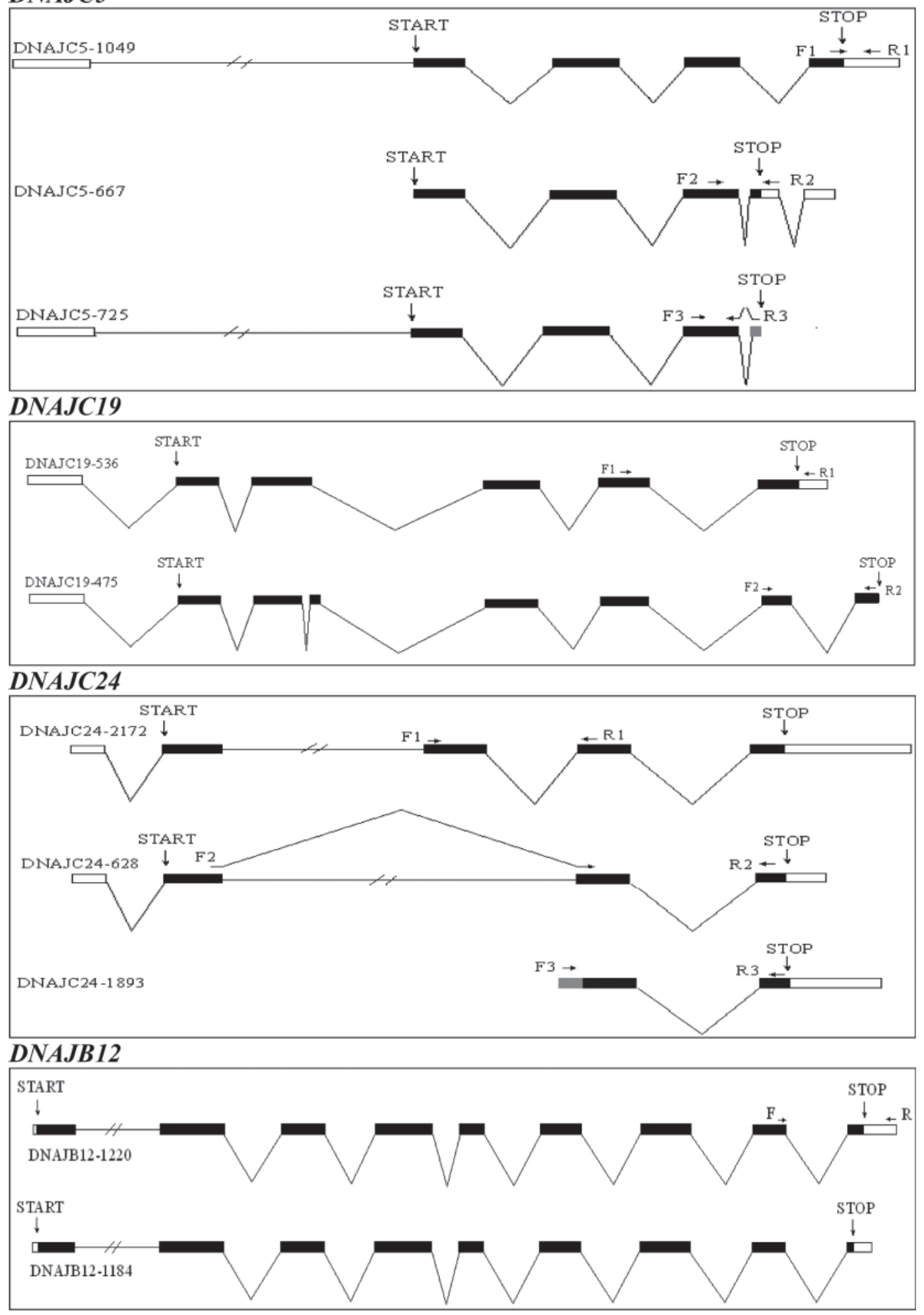

Figure 2. Splice variants of DNAJC5, DNAJC19, DNAJC24, and DNAJB12 genes. Positions of start and stop codons are indicated by vertical arrows and positions of primers used in the quantitative real-time PCR (qRT-PCR) are indicted by horizontal arrows. Black boxes represent coding sequences and white boxes represent untranslated regions. Due to overlap for DNAJB12, only transcript DNAB12-1220 could be amplified using transcript-specific primers. $\mathrm{F}=$ forward; $\mathrm{R}=$ reverse. 
Table 2. Blastocyst and fertilization rates for genotypic classes of DNAJC15 and DNAJC27 genes

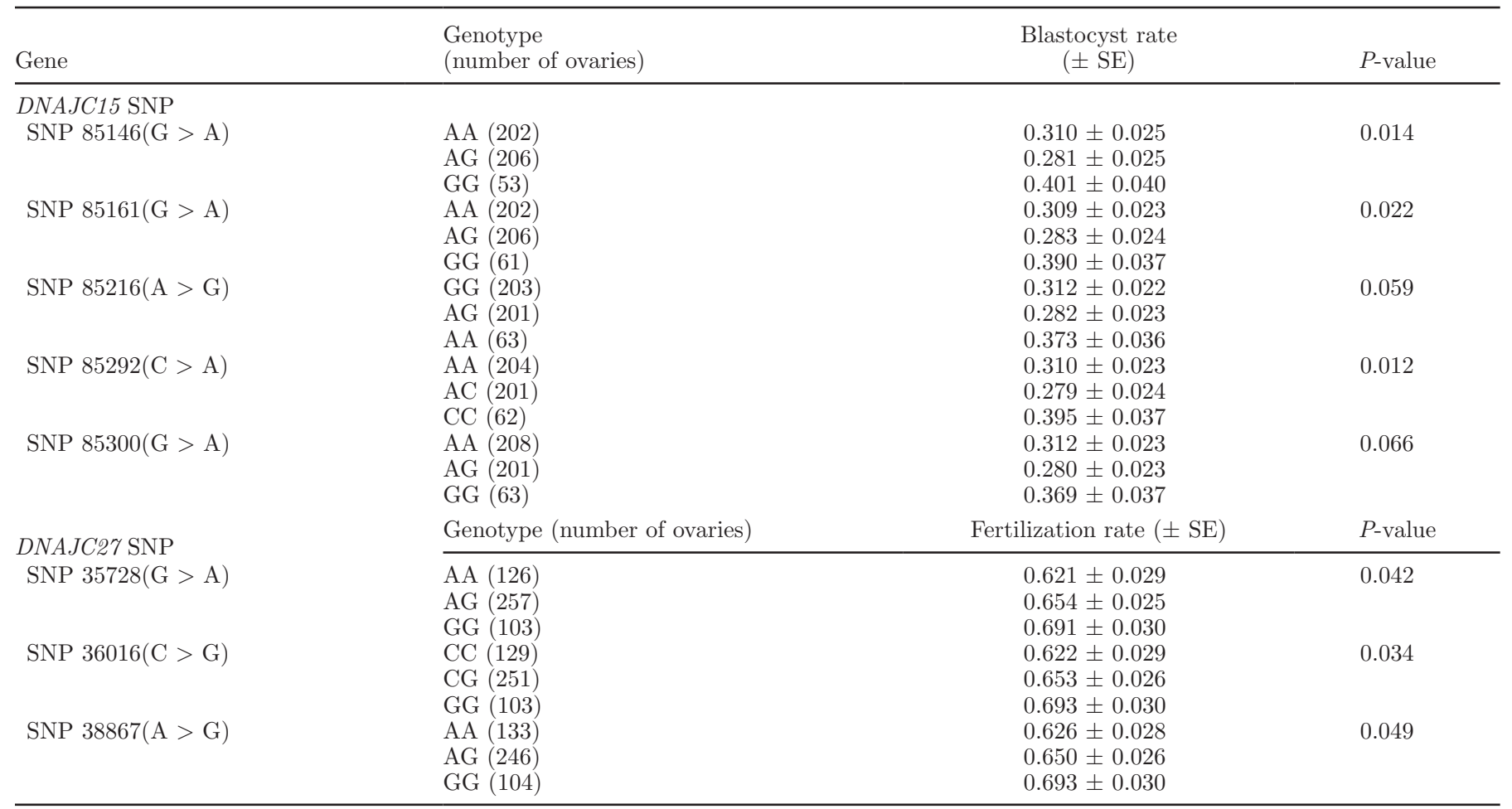

by interacting with key factors of the apoptotic signaling pathways (Lanneau et al., 2007). Therefore, it is feasible that upregulation of HSP40 genes observed in degenerate embryos is a response to stress on these embryos so they can maintain protein homeostasis.

The qRT-PCR analysis revealed moderate-fold change expression of HSPE1, HSPH1, HSB1, and 6 HSP70 genes. The HSP70 genes HSPA2, HSPA4, $H S P A 5$, and HSPA 8 showed higher expression in blastocysts than in degenerate embryos, whereas expression levels of HSPA9 and HSPA14 were higher in degenerate embryos. Although the specific functions of most HSP70 have not been reported in cattle, studies in humans and mice have indicated important roles for these genes in early embryogenesis. It has been reported that Hspa2 is essential for normal spermatogenesis and for growth and survival of cancer cells and that Hspas knockout mice are not viable because of the housekeeping functions of this gene (Daugaard et al., 2007). Human HSPA5 is involved in the folding and transport of proteins into the endoplasmic reticulum and Hspa 5 knockout mice embryos die at d 3.5, and therefore it, too, is considered a housekeeping gene (Daugaard et al., 2007). Furthermore, Matwee et al. (2001) have reported a decreased blastocyst rate and an increased apoptosis of embryos cultured in the presence of anti-HSP70 antibodies. Thus, upregulation and downregulation of
HSP in bovine embryos observed in the present study imply that these genes play vital roles in early embryo development.

Given that alternative splicing is a major source of phenotypic complexity in mammals (Wang et al., 2008), the Ensembl annotation of the bovine genome was searched and it was found that only 4 out of 17 HSP candidate genes have annotated splice variants. In contrast, for the 17 human and mouse counterparts, 15 and 13 genes, respectively, have 2 or more splice variants. The low number of alternative transcripts identified in cattle is presumably due to the incomplete annotation of the bovine genome. Indeed, in a previous study, thousands of alternative splicing events were characterized in blastocysts and degenerative embryos, and some of those events were found to be associated with the developmental status of embryos (Huang and Khatib, 2010).

To explore the effects of the different splice variants of HSP on embryo development, expression levels of the identified variants were estimated and compared between blastocysts and degenerate embryos. The results clearly show that different splice variants display different expression levels, which imply different functions in embryo development. For example, although the long variant of DNAJC24 was highly expressed in degenerate embryos compared with blastocysts, the short 


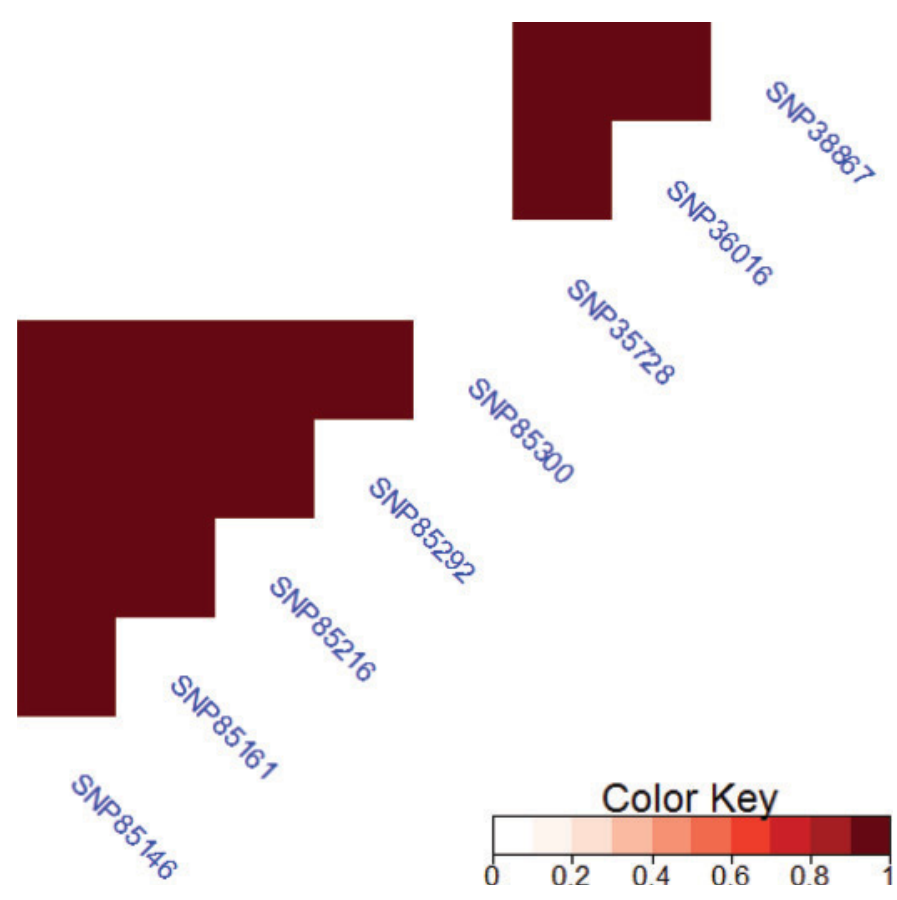

Figure 3. A heat map of linkage disequilibrium $\left(\mathrm{r}^{2}\right)$ between SNP in DNAJC15 and DNAJC2\%. Single nucleotide polymorphisms 85146, $85161,85216,85292$, and 85300 are located in DNAJC15, whereas SNP 35728,36016 , and 38867 are located in DNAJC27. Color version available in the online PDF.

variant of this gene was found to be lowly expressed in both embryo groups. Also, for DNAJC5, one transcript could not be detected using qRT-PCR. These results are consistent with human studies in which only some of the splice variants of genes were found to be associated with a disease state (Wang and Cooper, 2007). Collectively, these results testify to the importance of alternative splicing as a key regulator of phenotypic variation in mammals.

To better understand the involvement of the HSP genes in fertility traits, 4 genes that showed the most significant expression differences between embryo groups were tested for SNP association. Recently, an IVF experimental system in cattle has been developed in our laboratory (see Methods) aimed at identifying genetic factors affecting fertilization and embryo development. The effectiveness of this system in identifying genes and pathways associated with developmental and fertility traits has been demonstrated in several studies (Khatib et al., 2008a,b, 2009; Driver et al., 2009; Wang et al., 2009; Huang et al., 2010a). In this study, analysis of DNAJC15 and DNAJC27 SNP revealed significant associations with blastocyst and fertilization rates, respectively. These results are of particular significance because these 2 genes showed a remarkable difference in expression between blastocysts and degenerate em- bryos, providing strong evidence for the involvement of these genes in embryo production and development in cattle. These results also provide further evidence for the roles of HSP in the fertilization process and blastocyst rates observed in mice and cattle in previous studies. Neuer et al. (1998) found that the presence of anti-HSP60 monoclonal antibodies had significant effects on blastocyst rates of mouse embryos. Matwee et al. (2001) reported that the addition of anti-HSP70 monoclonal antibodies to the culture medium decreased the mean number of spermatozoa bound to zona pellucida by about $50 \%$ compared to that of controls. Also, the authors reported that anti-HSP70 antibodies significantly decreased the number of bovine embryos that reached the blastocyst stage (Matwee et al., 2001). A recent study by Rosenkrans et al. (2010) reported an association between SNP in the promoter region of HSP70 and calving percentages in Brahman cows. Although a small sample size was used in the association analysis, the results point to an important role of HSP70 in cow fertility.

\section{CONCLUSIONS}

The identification of specific HSP genes contributing to embryo survival can be a unique opportunity to improve protection of IVF embryos from different toxic conditions and to enhance pregnancy rates in cattle (Hansen, 2007). The HSP genes investigated in this study were found to be differentially expressed between blastocysts and degenerate embryos and SNP in 2 genes were associated with fertility traits. Blastocysts and degenerate embryos have distinct morphological and developmental features. As such, the present study provides a set of candidate genetic markers for preimplantation embryo development. The question whether the expression differences between blastocysts and degenerate embryos observed in this study have significant effects on development necessitates further research. However, it has been reported that relatively small changes (decrease or increase) in expression levels of HSP can result in growth abnormalities and cell death (Nollen and Morimoto, 2002). Also, the assessment of the expression of alternative transcript isoforms in the embryos examined in this study testifies to the importance of inclusion of this approach in studying gene expression.

\section{ACKNOWLEDGMENTS}

This study was supported by USDA Hatch grant WIS-142-PRJ17PH from the University of Wisconsin-Madison. Bao Zhang was supported by the China Scholarship Council (Beijing). 


\section{REFERENCES}

Al-Katanani, Y. M., and P. J. Hansen. 2002. Induced thermotolerance in bovine two-cell embryos and the role of heat shock protein 70 in embryonic development. Mol. Reprod. Dev. 62:174-180.

Becker, J., and E. A. Craig. 1994. Heat-shock proteins as molecular chaperones. Eur. J. Biochem. 219:11-23.

Blencowe, B. J. 2006. Alternative splicing: New insights from global analyses. Cell 126:37-47.

Daugaard, M., M. Rohde, and M. Jäättelä. 2007. The heat shock protein 70 family: Highly homologous proteins with overlapping and distinct functions. FEBS Lett. 581:3702-3710.

Dobson, H., R. Smith, M. Royal, Ch. Knight, and I. Sheldon. 2007. The high-producing dairy cow and its reproductive performance. Reprod. Domest. Anim. 42(Suppl. 2):17-23.

Driver, A. M., W. Huang, S. Gajic, R. L. Monson, G. J. Rosa, and H. Khatib. 2009. Effects of the progesterone receptor variants on fertility traits in cattle. J. Dairy Sci. 92:4082-4085.

Gotoh, T., K. Terada, S. Oyadomari, and M. Mori. 2004. hsp70-DnaJ chaperone pair prevents nitric oxide- and CHOP-induced apoptosis by inhibiting translocation of Bax to mitochondria. Cell Death Differ. 11:390-402.

Hansen, P. J. 2007. To be or not to be-Determinants of embryonic survival following heat shock. Theriogenology 68(Suppl. 1):S40 S48.

Huang, W., and H. Khatib. 2010. Comparison of transcriptomic landscapes of bovine embryos using RNA-Seq. BMC Genomics 11:711.

Huang, W., B. W. Kirkpatrick, G. J. Rosa, and H. Khatib. 2010a A genome-wide association study using selective DNA pooling identifies candidate markers for fertility in Holstein cattle. Anim. Genet. 41:570-578.

Huang, W., B. S. Yandell, and H. Khatib. 2010b. Transcriptomic profiling of bovine IVF embryos revealed candidate genes and pathways involved in early embryonic development. BMC Genomics 11:23.

Khatib, H., W. Huang, X. Wang, A. H. Tran, A. B. Bindrim, V. Schutzkus, R. L. Monson, and B. S. Yandell. 2009. Single gene and gene interaction effects on fertilization and embryonic survival rates in cattle. J. Dairy Sci. 92:2238-2247.

Khatib, H., C. Maltecca, R. L. Monson, V. Schutzkus, X. Wang, and J. J. Rutledge. 2008a. The fibroblast growth factor 2 gene is associated with embryonic mortality in cattle. J. Anim. Sci. 86:2063-2067.

Khatib, H., R. L. Monson, V. Schutzkus, D. M. Kohl, G. J. Rosa, and J. J. Rutledge. 2008b. Mutations in the STAT5A gene are associated with embryonic survival and milk composition in cattle. J. Dairy Sci. 91:784-793.

Lanneau, D., A. de Thonel, S. Maurel, C. Didelot, and C. Garrido. 2007. Apoptosis versus cell differentiation: Role of heat shock proteins HSP90, HSP70 and HSP27. Prion 1:53-60.

Leroy, J. L. M. R., G. Opsomer, A. Van Soom, I. G. F. Goovaerts, and P. E. J. Bols. 2008. Reduced fertility in high-yielding dairy cows: Are the oocyte and embryo in danger? Part I. The importance of negative energy balance and altered corpus luteum function to the reduction of oocyte and embryo quality in high-yielding dairy cows. Reprod. Domest. Anim. 43:612-622.
Livak, K. J., and T. D. Schmittgen. 2001. Analysis of relative gene expression data using real-time quantitative PCR and the $2^{-\Delta \Delta C T}$ method. Methods 25:402-408

Luft, J. C., and D. J. Dix. 1999. Hsp70 expression and function during embryogenesis. Cell Stress Chaperones 4:162-170.

Matwee, C., M. Kamaruddin, D. H. Betts, P. K. Basrur, and W. A King. 2001. The effects of antibodies to heat shock protein 70 in fertilization and embryo development. Mol. Hum. Reprod. $7: 829-837$.

Morris, D., and M. Diskin. 2008. Effect of progesterone on embryo survival. Animal 2:1112-1119.

Neuer, A., C. Mele, H. C. Liu, Z. Rosenwaks, and S. S. Witkin. 1998. Monoclonal antibodies to mammalian heat shock proteins impair mouse embryo development in vitro. Hum. Reprod. 13:987-990.

Neuer, A., S. D. Spandorfer, P. Giraldo, J. Jeremias, S. Dieterle, I Korneeva, H.-C. Liu, Z. Rosenwaks, and S. S. Witkin. 1999. Heat shock protein expression during gametogenesis and embryogenesis. Infect. Dis. Obstet. Gynecol. 7:10-16.

Nollen, E. A. A., and R. I. Morimoto. 2002. Chaperoning signaling pathways: Molecular chaperones as stress-sensing 'heat shock' proteins. J. Cell Sci. 115:2809-2816.

Parrish, J. J., A. Krogenaes, and J. L. Susko-Parrish. 1995. Effect of bovine sperm separation by either swim-up or Percoll method on success of in vitro fertilization and early embryonic development. Theriogenology 44:859-869.

Qiu, X.-B., Y.-M. Shao, S. Miao, and L. Wang. 2006. The diversity of the DnaJ/Hsp40 family, the crucial partners for Hsp70 chaperones. Cell. Mol. Life Sci. 63:2560-2570.

R Development Core Team. 2009. R: A Language and Environment for Statistical Computing. R Foundation for Statistical Computing, Vienna, Austria.

Rosenkrans, C. Jr., A. Banks, S. Reiter, and M. Looper. 2010. Calving traits of crossbred Brahman cows are associated with Heat Shock Protein 70 genetic polymorphisms. Anim. Reprod. Sci. 119:178-182.

Sambrook, J., and D. W. Russell. 2001. Molecular Cloning: A Laboratory Manual. 3rd ed. Cold Spring Harbor Laboratory Press, New York, NY

Santos, J. E. P., W. W. Thatcher, R. C. Chebel, R. L. A. Cerri, and K. N. Galvão. 2004. The effect of embryonic death rates in cattle on the efficacy of estrus synchronization programs. Anim. Reprod. Sci. 82-83:513-535.

Wang, E. T., R. Sandberg, S. Luo, I. Khrebtukova, L. Zhang, C. Mayr, S. F. Kingsmore, G. P. Schroth, and C. B. Burge. 2008. Alternative isoform regulation in human tissue transcriptomes. Nature 456:470-476.

Wang, G. S., and T. A. Cooper. 2007. Splicing in disease: Disruption of the splicing code and the decoding machinery. Nat. Rev. Genet. 8:749-761.

Wang, X., V. Schutzkus, W. Huang, G. J. Rosa, and H. Khatib. 2009. Analysis of segregation distortion and association of the bovine FGF2 with fertilization rate and early embryonic survival. Anim. Genet. 40:722-728. 